\title{
International repatriation following overseas
} disasters

\author{
N. J. JEFFERIES, C. RAMAGE \& A. BRISTOW \\ Department of Anaesthetics, St Bartholomew's Hospital, West Smithfield, London EC1A \\ $7 B E$
}

\section{SUMMARY}

The repatriation of 33 hospitalized patients to the United Kingdom following the Joigny coach accident in 1990 is described. The repatriation was undertaken by medical staff from St Bartholomew's Hospital Careflight project and EuropAssistance using a chartered McDonnell Douglas 83 aircraft. All patients were repatriated without mishap, but a number of difficulties were encountered.

It is recommended that agreement is reached in advance as to the organization that should handle overseas disasters involving British citizens. The organization should have expertise in repatriation as well as close ties with the NHS. A protocoB should be designed and adhered to. The initial response should involve despatching a team to the disaster country and provision of a control centre in the U.K. Special arrangements need to be made for staff and equipment. Liason with the airlines and ambulance services is essential.

\section{INTRODUCTION}

The St Bartholomew's Hospital Careflight project was set up to provide a national service for the transfer of critically ill patients between hospitals. Using a purposeequipped Aerospatiale twin Squirrel helicopter fitted as a one bed intensive care unit and permanently staffed by anaesthetic fellows and flight medical technicians, patients who need to be transferred can be moved between any two hospitals in the U.K. This has been described in full elsewhere (Kee, 1989; Ramage 1990).

Careflight also has access to both HS 125 and Beech 200 fixed wing aircraft which can be similarly equipped and staffed to transfer critically ill patients to and from other countries. A combination of helicopter and aircraft allows continual bedside to bedside monitoring and management to the standard normally expected on an ITU.

Correspondence: $\operatorname{Dr}$ A. Bristow, Department of Anaesthetics, St Bartholomew's Hospital, West Smithfield, London EC1A $7 B E$ 
During 1990, the project has been responsible for the medical services of EuropAssistance, a company that provides aid to travellers abroad. This paper describes the work undertaken by Careflight and EuropAssistance following the French coach crash of 3rd June 1990.

\section{CASE REPORT}

\section{The accident}

The tourist coach accident at Joigny, southwest of Paris, left 11 British tourists dead and 56 injured. Initial medical care was provided by the French emergency services, and patients were taken to three hospitals. There was a $24 \mathrm{~h}$ delay because the travel company could not be contacted to identify their medical insurance and thus the medical repatriation provider, following which EuropAssistance and Careflight assumed responsibility for repatriation of the victims.

\section{Initial organization}

Medical reports were collected by the British Consulate and sent to a control centre set up in London. The centre organized the repatriation and provided a $24 \mathrm{~h}$ support and information service for relatives. Medical reports were updated daily, and it was decided to repatriate 33 hospitalized patients and their relatives on 7 th June 1990. Three other patients were excluded because the severity and instability of their condition would have compromised the other patients. They were later repatriated by air ambulance. The remaining tourists returned home unaided.

\section{Patients}

All the casualties came from the Midlands. Their ages ranged from 9-76 years. Twenty-four were ambulant having sustained lacerations, abrasions and upper limb injuries. The nine stretcher cases included three chest injuries (two with chest drains, one with a thoracic epidural), three lower limb injuries, one fractured pelvis and two patients who had had laparotomies.

\section{The transfer}

Final medical summaries were faxed to London and reviewed to confirm suitability to travel. Ambulant and stretcher patients were correctly identified to prevent exceeding the aircraft's stretcher capacity.

The French office of EuropAssistance provided the doctors and nurses for the French part of the repatriation. The senior Careflight doctor participated in the morning briefing when drivers and medical attendants were allocated to individual patients.

All patients were reassessed to ensure accuracy of the reports. Appropriate 
monitoring was connected and analgesia administered. One patient required further management for bronchospasm. Stretcher patients were transported in individual ambulances, whilst ambulant patients, relatives and luggage were conveyed in a series of vans under police escort.

A McDonnell-Douglas 83 aircraft had been chartered for the transfer. It was reconfigured by folding down the seats on one side of the aisle and setting stretcher supports over them. A rear door was a particular advantage for loading the stretchers whilst other patients boarded by the forward side entrance.

Further Careflight staff met the convoy at thie aircraft. Boarding was undertaken in an order laid down the previous day so as to ensure ease of embarkation in the U.K.

On arrival at Birmingham airport, the aircraft was met by Careflight doctors and nurses who had already been allocated their patients and briefed on their condition. The French team handed over their patients, and patients were disembarked in order under the direction of the senior doctor. The Careflight Aviation Director coordinated personnel at the airport and organized the ambulances to ensure they arrived at the aircraft in the correct order.

The walking wounded who did not need further hospitalization were taken to their homes by coach. They were given their medical reports and X-rays and instructed to contact their GPs the following day. The GPs were also contacted by the medical desk in London to ensure continuity of treatment.

NHS ambulances were provided by the West Midlands Ambulance Service. Stretchers were loaded into a 'Jumbulance', a large vehicle resembling two coaches with a flexible joint in the middle. Access is by a front door and a one metres square lifting platform at one side. It was considered impractical to load one $80 \mathrm{Kg}$ patient with rib fractures and a chest drain, so a separate ambulance had to be called.

The patients who required hospitalization were delivered to a total of five hospitals. The nearest suitable hospital to their home had been identified and the admissions arranged by telephone in advance.

\section{DISCUSSION}

Overseas accidents that involve significant numbers of British citizens require specific planning and management. The problems encountered with major incidents in the U.K. will be compounded by distance and language difficulties. In this incident, the quality of medical care available locally was at least as good as that afforded in the U.K., but an incident in another country may demand the support of British medical teams for initial treatment as well as repatriation.

Using the experience of this incident, a number of recommendations can be made. Firstly, there must be a prompt and efficient initial response, which requires one organization to be responsible for such disasters. Difficulties in determining responsibility could be overcome if the insurance industry and the various repatriation companies agreed that one organization would deal with all such incidents 
without waiting to obtain policy details and without risk of financial loss which otherwise could extend to over $£ 100000$ :

Our normal initial response is to send an air ambulance with a medical team of three and a coordinator. If necessary, this can normally be airborne within $10 \mathrm{~min}$. The role of the team is to assess the extent of the disaster and provide the control centre in London with information. Further medical staff and equipment may then be despatched if the local medical services are insufficient. The aircraft can also repatriate two critical patients if local facilities are inadequate.

EuropAssistance consists of several separate organizations in different countries. In this incident, the Paris office had told their counterparts in London that they would manage the repatriation. Subsequently they decided to only provide doctors and nurses from France to the U.K. airport. This demonstrated the dangers of not following the normal protocols. As a result there was a need to hand over patients from one team to another in the aircraft as well as a need for extra vigilance to ensure there were no administrative omissions.

It is important to have one doctor in charge of the disaster, who should ideally be situated at the U.K. disaster desk. The actual repatriation should be under the control of another senior doctor on site abroad. This arrangement worked well in our case, and ensured that patients were properly accepted by hospitals or their general practitioners in advance.

Continuity of care is complicated by language difficulties and variations in drugs between countries. The coordinator, who should ideally speak both languages, is vital in arranging for medical reports, pathology results and X-rays to accompany the patients, as well as liasing with the land ambulances and organizing accommodation for the overseas team.

The amount of staff and equipment required will overwhelm most repatriation organizations. The medical and nursing staff must be qualified in emergency medicine as well as intensive care. They must have experience in dealing with patients in flight. Suitable clothing and prominent identification thereon is essential. Most of our patients were not seriously injured, but pulse oximetry and electrocardiography are needed for patients with chest injury, heart or lung disease, or autonomic disturbances (Frankel 1987; Ridley 1989). More seriously ill patients will require ventilation, gas analysis and invasive monitoring (Armitage, 1990).

We have equipment for up to six ventilated patients and eight additional monitored patients. If the casualties exceed our equipment capabilities further equipment can be requested from St Bartholomew's Hospital, and staffing logistics are handled in the same way. A formal link between the NHS and such repatriations not only assists with these difficulties but allows the provision of more specialist staff depending on the nature of the incidents. A national register of staff and equipment would be of value for larger incidents.

The type of aircraft will depend on distance and number of casualties. It is advantageous to discuss availability in advance with various airlines. The cabin will need to be reroled to accept the stretchers, and this can be undertaken more rapidly and efficiently if the airline has been involved in advance. The type of stretcher must be compatible with the aircraft. Patients should be placed on one 
stretcher for the entire repatriation to minimize the risk of pain and injury when changing stretcher.

The repatriation flight presents a number of areas for consideration. Food and drink will be needed for staff, patients and relatives. Patient access in flight may be more restricted than in a dedicated air ambulance. Simple stretcher bases may not allow patients to sit head up. Privacy for bedpans and other procedures can be overcome by using disposable surgical drapes with adhesive edges attached to the cabin ceiling.

The disembarkation in the U.K. is the time most prone to problems. In the past we have used private ambulance services, but found the lack of coordination and variety of equipment and staff unsatisfactory. The West Midlands Ambulance Service provided a much better solution, although the Jumbulance was not as satisfactory as individual ambulances. This needs to be discussed in advance to plan attending staff numbers and minimise journey times for the more seriously ill.

In summary, we recommend that proper consideration is given to dealing with overseas disasters in advance. Responsibility should be given to one organization which is expert in aviation medicine as well as having a good interface with the NHS. The input of the airlines, especially those such as British Airways who have their own disaster plans and medical staff is valuable. Protocols must be laid down and adhered to. The British ambulance services should also be involved in drawing up the protocols.

\section{ACKNOWLEDGEMENTS}

We would like to thank the French emergency services and British consular staff for their professionalism and untiring assistance. We are grateful to the operations staff at EuropAssistance for their help and support and the West Midlands ambulance service.

\section{REFERENCES}

Armitage J. N., Pyne A., Williams S. J., Frankel H. (1990) Respiratory problems of air travel in patients with spinal cord injuries. British Medical Journal 300, 1498-1499.

Frankel L. R. (1987) The evaluation, stabilisation and transport of the critically ill child. Critical Care Transport. International Anaesthiology Clinics 25, 77-103.

Kee S., Ramage C., Bristow A. (1989) The movement of the critically ill patient between hospitals. Care of the Critically Ill 5, 200-204.

Ramage C., Kee S., Bristow A. (1990) Interhospital transfer of the critically ill patient by helicopter. British Journal of Hospital Medicine 43, 147.

Ridley S. A. \& Carter R. The effects of secondary transport. Anaesthesia 44, 822-827. 